\title{
Adjustment of mobility parameters for traffic steering in multi-RAT multi-layer wireless networks
}

\author{
Pablo Muñoz ${ }^{1 *}$, Daniela Laselva², Raquel Barco ${ }^{2}$ and Preben Mogensen ${ }^{2,3}$
}

\begin{abstract}
Mobile broadband traffic has been increasing in the last years. To cope with such traffic demand, heterogeneous networks are an effective solution characterized by deploying networks with different cell sizes, radio access technologies (RATs) and carrier frequencies. Since the coverage area of these networks (or layers) is partially overlapped, users can be steered to a specific layer in order to improve network performance. In this work, two mechanisms of traffic steering are analyzed in a realistic high-speed packet access/long-term evolution deployment scenario, where the objective is to offload traffic from the macro-layer to pico-cells. In idle mode, i.e., the state in which no dedicated resources have been established for the user, a static adjustment of the layer priorities in the cell reselection algorithm is performed. In connected mode, where dedicated resources have been established, the parameters of the inter-RAT handover triggering condition are statically adjusted to offload the macro-layer. Simulation results show that further offloading can be achieved by modifying these algorithms so that user satisfaction is improved.
\end{abstract}

Keywords: Traffic steering, Heterogeneous networks, Handover, Cell reselection

\section{Introduction}

Heterogeneous networks (HetNets) will involve a diverse environment characterized by the presence of networks (layers) with different technologies, frequencies, cell sizes, etc. Future radio access technologies (RATs) such as long-term evolution (LTE) and LTE-advanced (LTE-A), which provide high data rates and good quality of service, will be deployed over the existing technologies such as Global System for Mobile Communications (GSM), Universal Mobile Telecommunications System (UMTS), high-speed packet access (HSPA) and WiFi. This need of extra capacity comes from the increasing complexity in the mobile terminals (presently called smartphones), the growing demand for online applications (such as video and music streaming services), and the increasing number of hotspots (small areas with high demand of traffic).

Both operators and users can select the preferred network layer among several candidates since the coverage

\footnotetext{
*Correspondence: pabloml@ic.uma.es

${ }^{1}$ Communications Engineering Department, University of Málaga, Malaga 29071, Spain

Full list of author information is available at the end of the article
}

area of those network layers is partially or totally overlapped in HetNets. This decision depends not only on the operator network deployment but also on the availability of technologies in the terminal. Each layer can also have a different cell size, which range from large sizes (macro-cells) to small sizes (pico- and femto-cells). Cells with smaller size are appropriate to provide higher capacity in areas with hotspots or remove coverage holes in urban areas. In addition, each network layer may operate at a different frequency that depends on the radio access technology and the radio spectrum license acquired by the operator.

The potential of steering a user connection towards a specific network layer in HetNets brings new challenges to the operators. This type of actions, known as traffic steering, attempts to use the resources in a more efficient way. Such a challenging task of selecting the best network due to the widespread deployment of overlapping wireless networks has been addressed in the literature. In [1], a policy-enabled handover $(\mathrm{HO})$ to express policies on what is the best wireless system is proposed. These policies allow to establish different trade-offs among indicators

\section{焦 Springer}

(c) 2013 Muñoz et al:- licensee Springer. This is an Open Access article distributed under the terms of the Creative Commons Attribution License (http://creativecommons.org/licenses/by/2.0), which permits unrestricted use, distribution, and reproduction in any medium, provided the original work is properly cited. 
related to cost, performance, power consumption, etc. In [2], an analytical approach to define a wide range of RAT selection policies, taking into account several allocation criteria such as service type and load, is proposed.

Users can also simultaneously use services through different RATs. Such a problem is addressed in [3,4], where different strategies in a multi-RAT, multi-cellular, and multi-service scenarios are designed to indicate the suitability of selecting a specific RAT. Similarly, the problem of allocating multiple services onto different subsystems in multi-access wireless systems is addressed in [5], where some principles for how this service allocation should be done to maximize the resulting combined capacity are discussed.

There are also many references focused on particular cases showing the benefits of traffic steering. For instance, a network layer suffering from a temporary traffic congestion can offload some users to a nonloaded layer (load balancing) [6,7]. High speed users can be connected to a so-called umbrella layer to avoid a frequent number of handovers [8]. Other factors contributing to the preferred choice of the access technology are addressed in [9], where aspects such as coverage, securing QoS for the requested service, minimizing the cost of delivering the service to the end user and maximizing the spectrum utilization by traffic packaging are considered to select the access technology. Potentials of dynamic traffic steering algorithms are also addressed in [10].

As shown in previous references, the specific objectives of traffic steering depend on operator policy, and they are achieved by utilizing mechanisms of mobility management, which are usually standardized by organizations such as the 3rd Generation Partnership Project (3GPP) [11]. In idle mode, no dedicated resources have been established for the user equipment (UE), and the procedure for changing of camping cell is called cell reselection. A traffic steering policy can be used in this case to determine the preferred layer for camping $[3,12]$. In connected mode, an $\mathrm{HO}$ is typically carried out when the UE moves between two cells to preserve the user connection. However, this is not necessarily the only reason for triggering an HO. Since the coverage area of the layers is overlapped, a UE could trigger an $\mathrm{HO}$ due to traffic steering reasons $[1,13]$. An important issue that arises from using both cell reselection and $\mathrm{HO}$ procedures for traffic steering is the need of aligning these two mechanism to avoid ping-pong effects.

Most of the previous references are focused on selecting the preferred RAT disregarding the fact that heterogeneity of future networks also includes networks with different frequencies, cell sizes, etc. In addition, the RATs used as examples for assessing the traffic steering techniques typically have been GSM, UMTS, and WLAN so that further study on future networks such as LTE and LTE-A would be necessary.

This paper investigates traffic steering techniques by static adjustment of mobility parameters in HetNets. To find the optimal user distribution across the network layers, a cell reselection algorithm considering different layer priorities and inter-RAT HOs is used for traffic steering purposes. More specifically, first, the problem of allocating absolute priorities (APs) to network layers for cell reselection is analyzed. Second, a procedure to select the optimal thresholds of the 3GPP events that trigger the inter-RAT HOs is proposed. In this case, the problem arising from the use of $\mathrm{HO}$ triggering events based on the signal level is addressed. When the events are based on the signal level instead of the signal quality, the thresholds involved in these events require a more precise adjustment, especially in multi-layer scenarios, where the location of low-power nodes within the macro-cell may determine the adjustment of the thresholds. The proposed techniques are assessed in a realistic scenario thoroughly deployed to cover hotspots with LTE pico-cells so that the traffic steering policy is to offload traffic to the picolayers in order to use more efficiently the radio resources and improve service performance. The main contribution of this work is the design of an algorithm to simplify the task of adjusting these thresholds for traffic steering. The impact of the LTE penetration in the mobile terminals is also studied in the LTE/HSPA scenario, where the LTE pico-cells are deployed in areas without LTE macro-coverage.

The rest of the paper is organized as follows. Section 2 is an overview of the deployment scenario and the mobility management algorithms used for traffic steering. Section 3 describes the procedure for static adjustment of mobility parameters for traffic steering purposes. Section 4 discusses the simulation results, and Section 5 presents the main conclusions of the study.

\section{Overview of mobility management}

\subsection{Deployment scenario}

A key aspect to select the deployment scenario is the heterogeneity expressed by the presence of different RATs, cell sizes, frequencies, hotspots, etc. in the network. However, any combination of these factors is not always a relevant deployment so that the scenario must be also realistic. For these reasons, the multi-RAT macro-pico scenario described in Figure 1 has been adopted in this work. Such a scenario is also representative of multiple challenges arising from HetNet scenarios, as described in the following paragraphs.

The deployment scenario is composed of four network layers, each of which comprises all the cells of the same characteristics. In this work, a network layer is given by a certain frequency carrier, RAT, and cell type (referring 


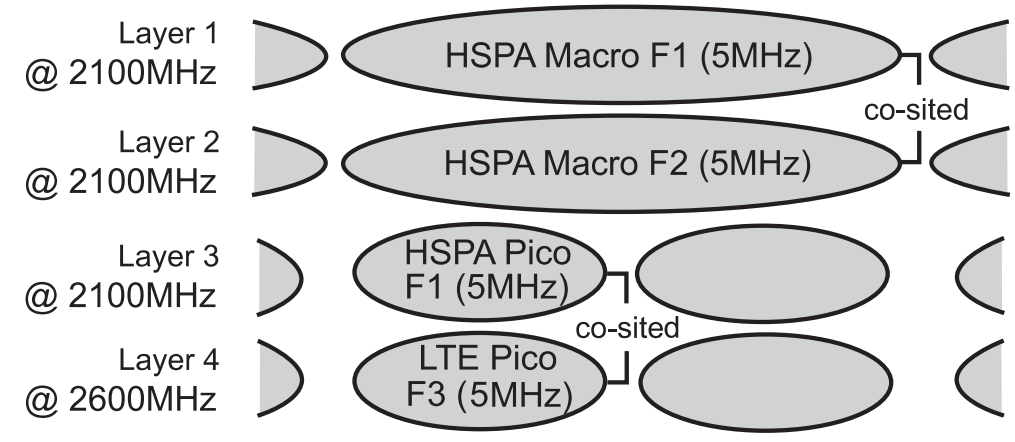

Figure 1 Deployment scenario.

to the cell size). An HSPA escape carrier, F2, has been included as another macro-layer, named layer 2, which is free of interference from other layers, and it is typically used by operators to provide additional capacity to macro-users [14]. These two layers are co-sited (i.e., base stations are located in the same position). The presence of hotspots forces the operators to provide more capacity in those areas by deploying cells of smaller sizes. Therefore, in the deployment scenario, LTE and HSPA pico-layers have been deployed in areas with hotspots, and these two layers are also co-sited. This configuration could be used in an early stage of LTE deployment in which LTE pico-cells have been deployed initially without LTE macro-coverage. In addition, there is no picocell clustering in the deployment scenario, i.e., pico-cells are far enough away from each other. This means that traffic steering between non-co-sited pico-cells cannot be properly performed in this scenario. Another feature drawn from Figure 1 is that layers 1 and 3 are cochannel interferers. As a result, the interference induced by the co-channel layer would be large in the serving cell edge.

In the deployment scenario, mobility management is based on algorithms standardized by the 3GPP. Intra- $/$ inter- RAT cases and intra-/inter-frequency cases when the user changes of cell are all covered in [15-17]. The following paragraphs describe the mobility management algorithms used in this work for traffic steering.

\subsection{AP-based cell reselection algorithm}

In idle mode, the UE reselects a new cell to camp on it if the cell reselection criteria is fulfilled. Traditionally, the cell-ranking criterion $R$ and the cell selection criterion $S$ have been evaluated in this process [15]. However, in a context of different frequencies and RATs, AP may be provided to the UE for cell reselection.

APs is a feature that allows the operator to prioritize inter-frequency and inter-RAT network layers during the cell reselection process. Firstly, the UE finds candidate cells by checking criterion $S$ :

$$
S_{\text {rxlev }}=Q_{\text {rxlevmeas }}-Q_{\text {rxlevmin }}>0 \text {, }
$$

and

$$
S_{\text {qual }}=Q_{\text {qualmeas }}-Q_{\text {qualmin }}>0,
$$

where $Q_{\text {rxlevmeas }}$ and $Q_{\text {qualmeas }}$ are the measured signal level and signal quality, respectively, and $Q_{\text {rxlevmin }}$ and $Q_{\text {qualmin }}$ are the minimum required values for each of the previous measurements.

Once criterion $S$ is fulfilled, the following condition depends on the cell priority. If the target cell has higher priority than the serving cell, the condition to be satisfied is as follows:

$$
S_{\text {qual }, t}>\text { Thresh }_{X, \text { HighQ }},
$$

where Thresh ${ }_{X, \mathrm{HighQ}}$ is the threshold (in $\mathrm{dB}$ ) used by the UE on the target cell $t$ when reselecting towards a higher priority frequency or RAT. If the priority of the target cell is lower than or equal to the serving cell, then there are two conditions to be fulfilled:

$$
S_{\text {qual }, s}<\text { Thresh }_{\text {Serving,LowQ }},
$$

and

$$
S_{\text {qual }, t}>\text { Thresh }_{X, \text { LowQ }},
$$

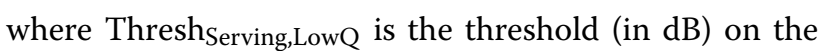
serving cell $s$ and Thresh $\mathrm{X}_{X, \mathrm{LowQ}}$ is the threshold on the target cell $t$. Note that the above conditions are referred to the signal quality, but they can be also based on the signal level. In LTE and HSPA, when absolute priority information for inter-RAT and inter-frequency cases is available, the AP-based cell reselection algorithm is used. The conditions to be applied between the different cases (i.e., HSPA to HSPA and HSPA to LTE) are the same, but the signal measurements are technology-dependent. When multiple cells of different priorities are suitable for reselection by fulfilling the above conditions, cells of 
higher priority will take precedence over a lower priority frequency or RAT. For intra-frequency and equal priority inter-frequency cases, criterion $R$ is applied. $R$ is a cellranking criterion based on either of signal quality or signal level.

An example of the cell reselection when APs are used in LTE is shown in Figure 2, where the signal quality received by a UE from two cells with higher/lower priority is drawn. As it can be observed, the UE firstly reselects towards a cell of the lower priority layer. After this, the UE returns to the initial layer. Note that the conditions shown in this section must be fulfilled during a time interval named $T_{\text {reselection. In Section 3, the cell reselection priority levels }}$ will be assigned to the network layers of the deployment scenario according to the traffic steering policy.

\subsection{Inter-RAT HO algorithm based on the $B 2 / 3 A$ events}

Inter-RAT HOs refer to those HOs carried out between cells from different RATs when the UE is in connected mode. An HO from LTE to HSPA is performed when the $B 2$ event is triggered, that is, the reference signal received power (RSRP) of the LTE serving cell $s$ becomes worse than the threshold $B 2 \_1$ and the received signal code power (RSCP) of the HSPA target cell $t$ becomes better than the threshold $B 2 \_2[16]$ :

$$
\operatorname{RSRP}_{s}<B 2 \_1 \text { and } \operatorname{RSCP}_{t}>B 2 \_2 \text {. }
$$

Similarly, an HO from HSPA to LTE is performed when the $3 A$ event is triggered [17]. In this case, the thresholds $3 A \_1$ and $3 A \_2$ are used for evaluation:

$$
\mathrm{RSCP}_{s}<3 A_{-} 1 \text { and } \mathrm{RSRP}_{t}>3 A_{-} 2 \text {. }
$$

The previous measurements performed by the UE are based on the signal level. However, such measurements can be also referred to the signal quality so that the reference signal received quality (RSRQ) and $\mathrm{CPICH}$ Ec/No are used for measuring LTE and HSPA cells, respectively. From the operator's perspective, working with observable measurements (e.g., the RSRP) is usually preferable since the concept of quality sometimes requires certain level of abstraction and complexity to draw inferences from the measurement analysis. For this reason, in this work, the measurement type for triggering $\mathrm{HO}$ events is based on the signal level instead of the signal quality. The main drawback is that the adjustment of the thresholds based on the signal level may depend on the specific network layer (e.g., the cell size). The next section discusses more about how to define these thresholds from traffic steering purposes.

\section{Mobility management for traffic steering}

A reasonable traffic steering strategy is to take full advantage of the existing pico-cells by offloading as much traffic as possible from the macro-cells. Since the pico-cells have been thoroughly located in hotspots, most of the traffic in hotspots are carried by the pico-layer. However, mobility management can be used to further offload traffic from macro-cells to this layer, assuming that there is still some capacity remaining in the pico-cells. Thus, some parameters of the mobility management algorithms in both idle and connected modes can be modified for this purpose.

\subsection{Traffic steering in idle mode}

In idle mode, further offloading to pico-cells can be achieved by simply changing the APs of the network layers used for cell reselection. The objective is to enforce such a user distribution across the layers that the resource utilization is optimal. Since the HO has a signaling cost and a data transferring cost, it is important to achieve the optimal user distribution from the idle mode. A full alignment of user distributions in both idle and connected modes also avoids the ping-pong effect between these two states.

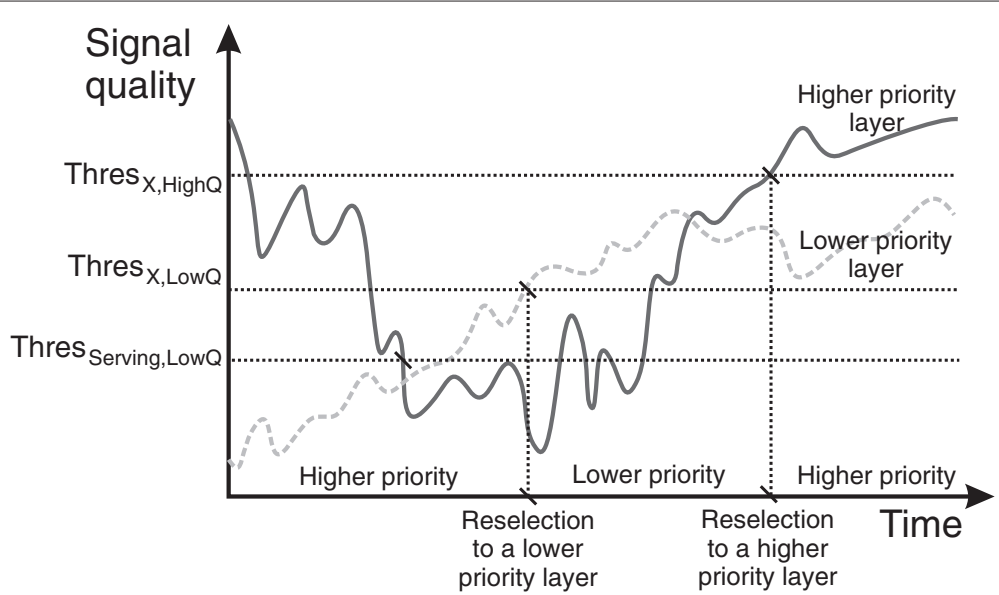

Figure 2 Example of the AP-based cell reselection. 
To allocate APs to networks layers, several considerations must be taken into account. First, those network layers belonging to the same RAT and sharing the same frequency must have the same AP. Thus, in the deployment scenario, layers 1 and 3 , which share frequency $F 1$, cannot have different AP levels [15]. Another limitation imposed by the 3GPP is that two RATs sharing the same priority level is not supported. In the deployment scenario, this implies that the pico-layers cannot have the same priority so that one layer takes higher priority for the macro-offloading. As layer 1 causes co-channel interference with layer 3 , the highest priority should be allocated to layer 4. Looking at the previous constraints together with the objective of offloading traffic from the macrolayers, two candidate sets of AP allocation are proposed. The difference lies in the relative priority between layers 1 and 2, that is, whether the macro-escape carrier takes priority over the main carrier or it is just the opposite. Thus, given that 0 is the lowest priority level, the two proposed sets of APs for the deployment scenario are as follows:

$$
\mathrm{AP}_{1}=[L 1, L 2, L 3, L 4]=[0,1,0,2] ; \mathrm{AP}_{2}=[1,0,1,2]
$$

where $L X$ refers to layer $X$. In both cases, it is expected that a user located in a hotspot has higher probability of starting a connection from the LTE layer, according to the traffic steering policy.

\subsection{Traffic steering in connected mode}

When the UE is in connected mode, traffic steering can be performed by forced HOs, cell barring, or adjusting $\mathrm{HO}$ parameters. The latter is the simplest mechanism because it does not need to be user-specific and it is not as rigid as cell barring. In addition, it is possible to define different thresholds for the same $\mathrm{HO}$ triggering event according to particular policies. For instance, radio-driven HOs are concerned with HOs carried out when the UE experiences poor signal quality, but HOs due to traffic steering purposes can be also defined. In the first case, UEs will be generally handed over to cells with better quality, by setting $B 2 / 3 A$ thresholds. Conversely, in the second case, UEs will be generally handed over under good signal conditions. Since these two cases are complementary, two different sets of thresholds for a specific $\mathrm{HO}$ triggering event can be defined in the network.

Traffic steering is performed here by adjusting the thresholds of the (inter-RAT) $B 2$ and $3 A$ events in LTE and HSPA, respectively. As mentioned before, in the deployment scenario, $L 4$ has been selected to offload traffic from macro-cells because it does not receive interference from any other layer. It is noted that offloading between picocells is not considered as pico-cells in the deployment scenario are typically far enough away from each other. In addition, the number of inter-RAT HOs between co-sited pico-cells (layers $L 3$ and $L 4$ ) is expected to be negligible because of the difficulty of fulfilling both conditions (for the serving and the target cell) in Equations 6 and 7. Thus, this study is only focused on macro-pico HOs, disregarding the pico-pico case. Note that, if a larger deployment of pico-cells were considered in the scenario, a certain hysteresis region should be considered when adjusting the $B 2 / 3 A$ thresholds in order to avoid ping-pong HOs between non-co-sited HSPA and LTE pico-cells due to user mobility.

A single set of $B 2 / 3 A$ thresholds defined in the network might be enough for traffic steering. However, it is also possible to have the thresholds defined per layer pair. The deployment scenario includes two macro-pico layer pairs as there is an additional macro-layer given by the HSPA escape carrier $(F 2)$. Unlike the HSPA main carrier $(F 1), F 2$ does not receive interference from any other layer. This means that the optimal $B 2 / 3 A$ thresholds should not necessarily be the same for these layers. In this work, $B 2 / 3 A$ thresholds are assumed to be defined per layer pair. The benefit derived from this consideration will be analyzed in Section 4.

Another assumption made in Section 2 is that event measurements are based on the signal level instead of the signal quality. A problem arises when the signal level is used for the HO triggering condition and the deployment scenario includes cells of different sizes. Suppose that a user $U_{1}$ is located in a hotspot $H_{1}$ and connected to the macro-layer, as shown in Figure 3. Due to the traffic steering policy, the thresholds $3 A \_1$ and $3 A \_2$ have been previously set so that $U_{1}$ is handed over to the neighboring LTE pico-cell. However, user $U_{2}$, located in $H_{2}$ (closer to the macro-cell base station), probably will not perform an $\mathrm{HO}$ because the condition involving $3 A \_1$ would not be fulfilled. Such a situation highlights that the adjustment of 3A_1 to offload the macro-cell depends on the pico-cell location. To avoid distance dependence, setting a very high value for $3 A_{-} 1$ is similar to the removal of the first condition in Equation 7. The same approach is applied to the case in which the UE is connected to LTE and is leaving a hotspot. To send the UE to the HSPA macro-cell, it is necessary to fulfill the second condition in Equation 6, regardless of the pico-cell location. Thus, setting a very low value for B2_2 would remove this dependence. However, this configuration is certainly dangerous as the UE could be handed over to a cell with very low signal level. For this reason, Algorithm 1 is proposed to adjust the thresholds of $B 2$ and $3 A$ events for traffic steering.

Algorithm 1 simplifies the problem to one parameter in order to determine the area where the UEs should connect to the pico-cell. Note that such a problem can be applied to any HetNet scenario in which the network deployment 


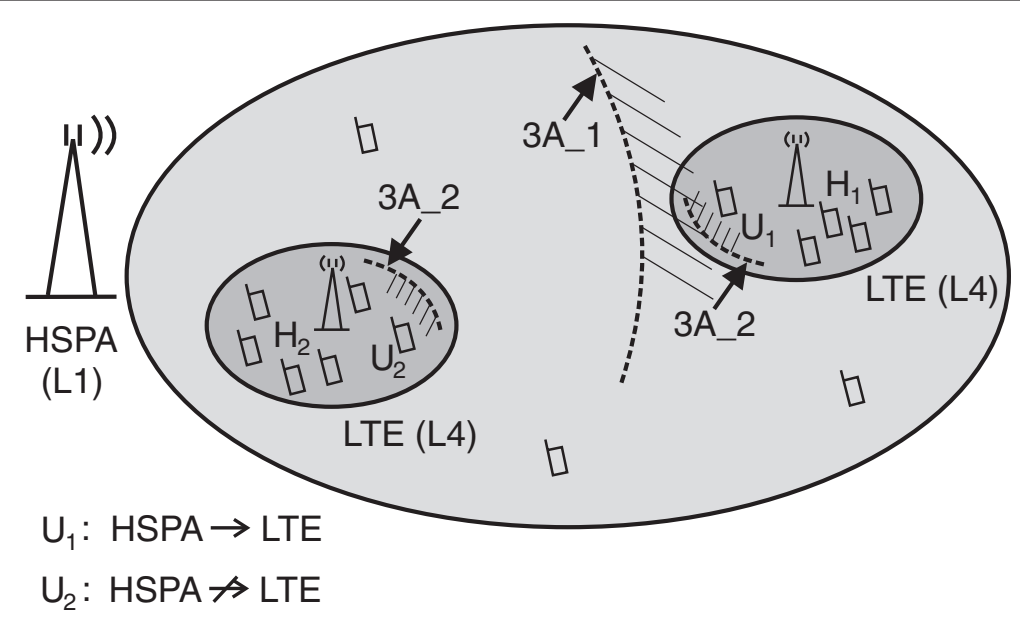

Figure 3 The impact of the measurement type in a multi-layer scenario.

comprises cells of different sizes and RATs. If $B 2 \_2$ and $3 A \_1$ have been set to be always fulfilled, $B 2 \_1$ and $3 A \_2$ can be used to define the concerned area in both directions, i.e., moving from LTE to HSPA and vice versa. In principle, these two parameters could have the same value, but a certain hysteresis $(\Delta)$ can be also applied to avoid the ping-pong effect between RATs. Step 2 consists of a sensitivity analysis of $B 2 \_1$ in order to select a value that optimizes the resource utilization. A set of values selected from the normal operating range of $B 2 \_1$ is assessed by looking at the main key performance indicators in order to find the optimal value, and then $B 2 \_1$ is set to this value. The remaining steps are devoted to optimize $B 2 \_2$ and $3 A \_1$. In the case of $B 2 \_2$, there may exist a trade-off between keeping the optimal resource utilization achieved in step 2 and avoiding low values which jeopardize the connection quality.

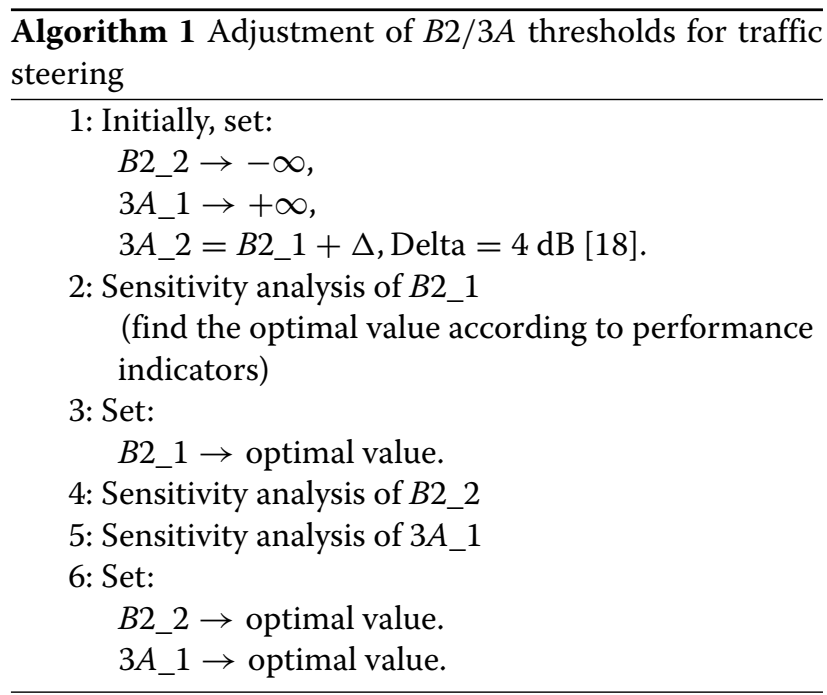

In real networks, context factors such as the system load are varied frequently and dynamically. To avoid that such variations affect the analysis involved in Algorithm 1, the performance indicators should be collected using a measurement period long enough. For example, as there are typically two distinguished traffic patterns within a day (i.e., daily and nightly traffic patterns), the measurement period could be the hours during the day. Thus, a new control parameter setting would be tested every day during the same time interval to ensure similar conditions. Note that this optimization process can last several weeks, which is the typical duration of field trial campaigns performed by operators and manufacturers. The optimal settings can then be fixed in the network during months or even years. In addition, Algorithm 1 can be executed periodically to cope with long-term changes in the traffic patterns, e.g., the center of a city becomes crowded and a new shopping center is built.

\section{Results}

\subsection{Simulation setup}

The performance of the proposed traffic steering techniques has been assessed with a dynamic system level simulator. Further details of the simulator implementation are found in [19]. The simulated scenario includes a macro-pico cellular environment where its macro-cell layout consists of seven regular hexagonal tri-sectorized sites and wraparound is assumed. The pico-cell layout is composed of two omni-directional sites per macro-area, randomly located, and covering a hotspot of similar size. A $68 \%$ traffic is generated and confined within hotspots. The service provided to the UEs is based on a realistic video streaming model, which generates traffic according to a source bit rate equal to $1,024 \mathrm{Kbps}$. Considering 
this, the offered traffic in the network is calculated as follows:

$$
\text { Offered traffic }=\frac{\mathrm{BR} \cdot \mathrm{SL}}{\overline{\mathrm{IAT}^{\prime}}}
$$

where $\mathrm{BR}$ is the service bit rate per session (in Mbps), $\mathrm{SL}$ is the session length (in seconds), and IAT' is the mean intercall time (in seconds) in the concerned area calculated as follows:

$$
\mathrm{IAT}^{\prime}=\frac{\text { IAT }}{N_{\text {users }}},
$$

where IAT is the mean inter-session time per mobile (in seconds) and $N_{\text {users }}$ is the number of mobiles in the concerned area. If the buffer at the UE is emptied, a rebuffering is needed and the video playout is interrupted. When the interruption time exceeds a specific threshold, the user is dropped out. The arrival rate of new connections is exponentially distributed. Only the downlink is considered in the simulation as it is the most restrictive link with this type of service in next-generation wireless networks. The main simulation parameters are summarized in Table 1. For each parameter setting of the sensitivity analysis, an independent simulation of $150 \mathrm{~s}$ in duration has been carried out. Note that this simulation time is large enough to obtain reliable measurements for the network size, the traffic model, and the system load used in this work. In addition, network conditions remain fairly constant throughout the simulations. This situation can emulate, e.g., the busy hour, the working hours in a business area, and the daytime in the downtown. If the system load varies dynamically throughout the day in the real network, firstly, the operator should identify the time frames in which the load remains fairly constant and then perform the optimization separately for each period. Alternatively, operators could set the parameters with the values optimized for the critical situation.

\subsection{Simulation results of traffic steering in idle mode}

The following allocations of APs are evaluated: $\mathrm{AP}_{0}=\left[\begin{array}{ll}0 & 0\end{array}\right.$

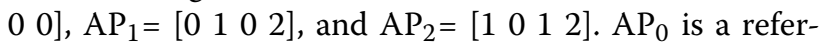
ence setting allocating the same AP to the network layers and included for comparison with the proposed settings $\mathrm{AP}_{1}$ and $\mathrm{AP}_{2}$. The settings of $B 2 / 3 A$ thresholds for interRAT HOs are the following: $B 2 \_1=-104 \mathrm{dBm}, B 2 \_=$ $-98 \mathrm{dBm}, 3 A \_1=-92 \mathrm{dBm}$, and 3A_2 $=-100 \mathrm{dBm}$. The reason for setting those values will be discussed in Section 4.3.

Figure 4 shows the total traffic carried per macro-cell area (i.e., two macro-cells and four pico-cells) as well as the traffic carried per network layer and macro-cell area. Note that in a macro-cell area, the offered traffic calculated using Equation 9 with the parameter values shown

\begin{tabular}{|c|c|}
\hline Parameter & Configuration \\
\hline System & HSPA, LTE \\
\hline \multirow[t]{3}{*}{ Layer } & HSPA: macro, $5 \mathrm{MHz}$ at 2,100 MHz (F1 and F2); \\
\hline & pico, $5 \mathrm{MHz}$ at 2,100 MHz (F1). \\
\hline & LTE: pico, $5 \mathrm{MHz}$ at 2,600 MHz (F3) \\
\hline \multirow[t]{3}{*}{ Cellular layout } & Macro: hexagonal grid, 21 cells ( $3 \times 19$ sites), \\
\hline & Inter-site distance $=0.5 \mathrm{~km}$. \\
\hline & $\mathrm{Pico}=2$ pico-cells/macro-area \\
\hline \multirow[t]{4}{*}{ Propagation } & Macro $137.6+34.8 \log _{10}(R)$ \\
\hline & [Hata-COST 231]; $43 \mathrm{dBm}$. \\
\hline & Pico $140.7+36.7 \log _{10}(R)$ \\
\hline & [3GPP TR 36.814]; 37 dBm. \\
\hline \multirow[t]{2}{*}{ Shadowing } & Gaussian, std 8 dB (macro), 10 dB (pico) \\
\hline & De-corr. length 50 m (macro), 13 m (pico) \\
\hline $\begin{array}{l}\text { Users } \\
\text { macro-area }\end{array}$ & 100 \\
\hline Users per hotspot & 34 (hotspot radius $=40 \mathrm{~m}$ ) \\
\hline User speed & Hotspot 3 km/h; Non-hotspot 3, 50 km/h \\
\hline Service model & Video streaming \\
\hline Bit rate & $1,024 \mathrm{Kbps}$ \\
\hline Mean inter-call time & $30 s$ \\
\hline Call duration & $10 \mathrm{~s}$ \\
\hline Dropping out & Interruption time $=10 \mathrm{~s}$ \\
\hline \multirow[t]{6}{*}{ Handover settings } & Intra-frequency A3 (RSRP / Ec/No, offset = $2 \mathrm{~dB}$ ), \\
\hline & A5 (RSRP/RSCP, A5_1 = -111 dBm, \\
\hline & $\left.A 5 \_2=-109 \mathrm{dBm}\right)$ \\
\hline & Inter-frequency $A 3(\mathrm{RSRQ} / \mathrm{Ec} / \mathrm{No}$, offset $=4 \mathrm{~dB})$ \\
\hline & Inter-RAT B2/3A (RSRP/RSCP) \\
\hline & Time to trigger $=0.5 \mathrm{~s}$ \\
\hline \multirow[t]{5}{*}{ Reselection settings } & Thresh $_{\text {Serving,LowQ }}=-13 \mathrm{~dB}$ \\
\hline & Thresh $_{X, \text { LowQ }}=-12 \mathrm{~dB}$ \\
\hline & Thresh $_{X, \text { HighQ }}=-10 \mathrm{~dB}$ \\
\hline & $Q_{\text {rxlevmin }}=-\operatorname{Inf}, Q_{\text {qualmin }}=-18 \mathrm{~dB}$ \\
\hline & $T_{\text {reselection }}=1.2 \mathrm{~s}$ \\
\hline Simulation time & $150 \mathrm{~s}$ \\
\hline Warm-up & $50 \mathrm{~s}$ \\
\hline $\begin{array}{l}\text { Simulation time } \\
\text { resolution }\end{array}$ & $100 \mathrm{~ms}$ \\
\hline
\end{tabular}

Table 1 Simulation parameters

in Table 1 is 33.333 Mbps, while in a hotspot, the offered traffic is $11.333 \mathrm{Mbps}$. It is observed that, although the layers have the same bandwidth (i.e., $5 \mathrm{MHz}$ ), the capacity is different for each one. This is because the system capacity depends on the user distribution and how the interference affects each layer. For instance, as users are more concentrated in the pico-cells, higher capacity for those cells 


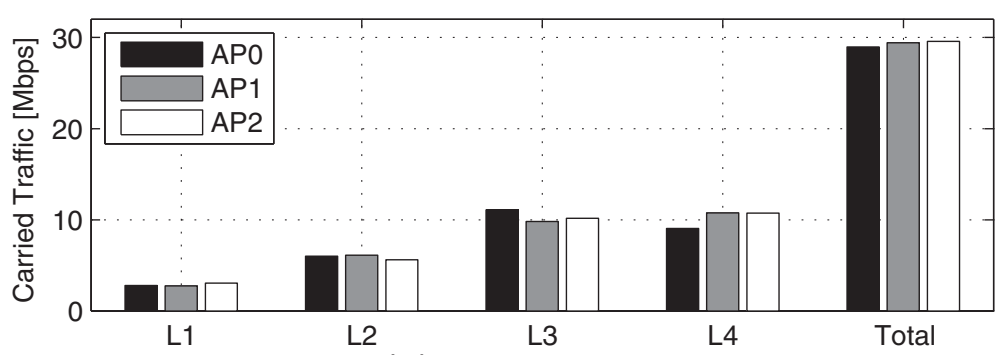

(a) $50 \%$ LTE penetration

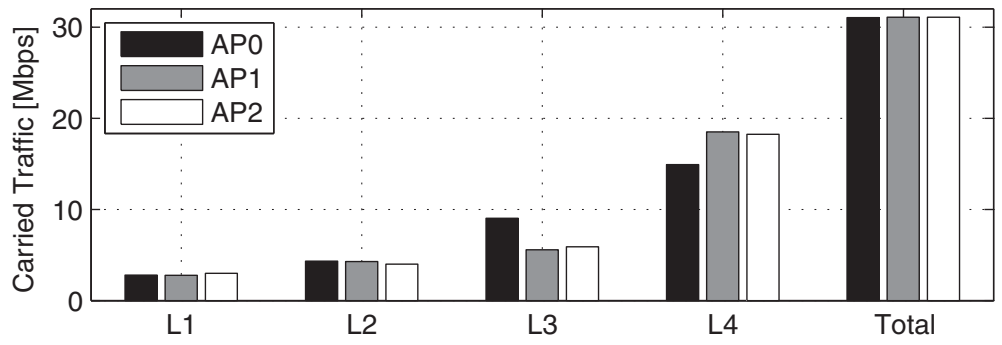

(b) $100 \%$ LTE penetration

Figure 4 Carried traffic per macro-cell area for different settings of APs. (a) 50\% LTE penetration. (b) 100\% LTE penetration.

(compared to macro-cells) is expected. In addition, as $L 1$ and $L 3$ are co-channel interferers, lower capacity for those layers (compared to $L 2$ and $L 4$ ) is expected.

Regarding the AP settings, with 50\% LTE penetration, more traffic (approximately $1.7 \mathrm{Mbps}$ ) is carried by $L 4$ when $\mathrm{AP}_{1}$ and $\mathrm{AP}_{2}$ are used. This is because further UE offloading to $L 4$ is achieved using the AP-based cell reselection. It is also noted that $\mathrm{AP}_{2}$ leads to a lower carried traffic in $L 2$ since the priority allocated to this layer is lower. In addition, $L 3$ and $L 4$ carry similar traffic volumes since the LTE penetration is only partial. Conversely, with $100 \%$ LTE penetration, $L 4$ carries considerably more traffic since there are more LTE-compatible UEs which can be transferred to $L 4$. In addition, more traffic (approximately $3 \mathrm{Mbps}$ ) is carried by $L 4$ when $\mathrm{AP}_{1}$ and $\mathrm{AP}_{2}$ are used instead of the reference case $\mathrm{AP}_{0}$. Note also that most of this traffic comes from the co-sited pico-layer (L3), which means that macro-offloading (i.e., traffic from macro- to pico-cells) is very limited.

On the other hand, regarding the total carried traffic in Figure 4, note that the differences between the AP settings are due to the dropped calls, which means that less traffic is carried by the network. The relationship between the carried traffic and the dropping ratio (i.e., the number of dropped calls to the total number of carried calls) is given by the following expression:

Carried traffic $=$ Offered traffic $\times(1-$ Dropping ratio $)$.
The dropping ratio is related in this paper to resource availability rather than to bad radio conditions, i.e., a lower value for this indicator means that enough resources are available to play out the video sessions with no or few interruptions. Thus, a better optimization of mobility parameters means a lower dropping ratio. The dropping ratio measured for the previous AP settings and LTE penetration levels is shown in Table 2. Note also that the dropping ratio is closely related to the blocking ratio. A user is blocked when it attempts to start a session but no resources are available. However, as the number of users in the simulation is fixed, all the users are accepted by the system (i.e., no measurements related to call blocking are performed). Thus, the benefits of reselection optimization will not impact on lower call blocking or better accessibility (i.e., higher number of served users) but on user satisfaction in terms of lower dropping ratio.

With 50\% LTE penetration, $\mathrm{AP}_{0}$ leads to lower total carried traffic since the dropping ratio is higher. This is because the traffic distribution within the pico-layers for $\mathrm{AP}_{0}$ is different from $\mathrm{AP}_{1}$ and $\mathrm{AP}_{2}$, as $\mathrm{AP}_{0}$ carries more traffic in $L 3$. Such a difference for the reference case causes higher interference to $L 1$, increasing the dropping ratio. With $100 \%$ LTE penetration, the dropping ratio is decreased as a higher number of UEs implementing LTE can be handed over from macro-cells to pico-cells. However, the difference in total carried traffic (and dropping ratio) between the three settings is negligible as the two pico-layers start to be overloaded when $100 \%$ LTE penetration is applied to the scenario 
Table 2 Key performance indicators for different AP settings in idle mode

\begin{tabular}{lcccccc}
\hline $\begin{array}{l}\text { Configuration } \\
\text { AP }\end{array}$ & LTE penetration (\%) & nRes/s & nHO/s & $\begin{array}{c}\text { HO PP } \\
\text { ratio }\end{array}$ & $\begin{array}{c}\text { RLF } \\
\text { ratio }\end{array}$ & $\begin{array}{c}\text { Dropping } \\
\text { ratio }\end{array}$ \\
\hline AP_0 & 50 & 102.7 & 31.6 & 7.2 & 4.3 & 15.0 \\
AP_1 & 50 & 122.1 & 31.8 & 6.5 & 4.1 & 13.5 \\
AP_2 & 50 & 126.3 & 30.6 & 6.5 & 4.5 & 13.2 \\
AP_0 & 100 & 137.1 & 38.6 & 10.0 & 3.0 & 3.2 \\
AP_1 & 100 & 189.8 & 36.8 & 9.0 & 3.1 \\
AP_2 & 100 & 194.4 & 35.9 & 9.7 & 3.0 & 6.1 \\
\hline
\end{tabular}

Total number of UEs $=2,100$. $\mathrm{nRes} / \mathrm{s}$, number of reselections per second; $\mathrm{nHO} / \mathrm{s}$, number of HOs per second; $\mathrm{HO} \mathrm{PP}$ ratio, $\mathrm{HO}$ ping-pong ratio; $\mathrm{RLF}$ ratio, radio link failure ratio.

(macro-offloading is higher). This effect is drawn from the resource utilization (percentage of the total amount of resources that are being used) depicted in Figure 5, where it is shown that the resource utilization in $L 4$ is approximately 50\% with 50\% LTE penetration and approximately $80 \%$ with $100 \%$ LTE penetration (i.e., approximately $30 \%$ increased). With $50 \%$ LTE penetration, the increased dropping ratio is due in part to an excessive resource utilization (99\%) in $L 2$. In this case, the limited macro-offloading performed by settings $\mathrm{AP}_{1}$ and $\mathrm{AP}_{2}$ increases the resource utilization in
L4 by only approximately $6 \%$. With $100 \%$ LTE penetration, this value reaches approximately $9 \%$. In addition, the resource utilization in $L 3$ is decreased by approximately $12 \%$.

The previous analysis shows that, in general, the user distribution between two layer pairs with coverage areas that are fully overlapped (e.g., co-sited layers) can be effectively modified by allocating different APs. In this case, APs can be used, for example, to reduce the interference generated over other intra-frequency layers by decreasing the percentage of UEs attached to the interfering layer

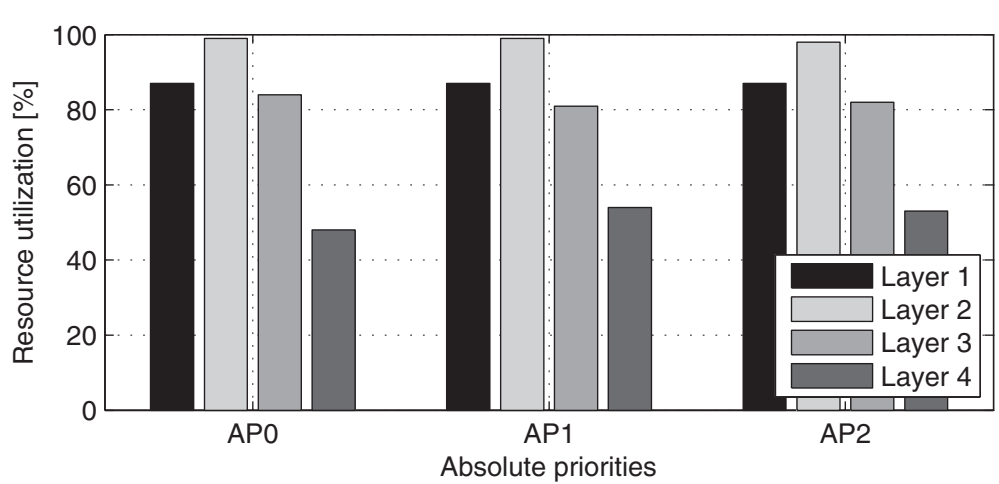

(a) $50 \%$ LTE penetration

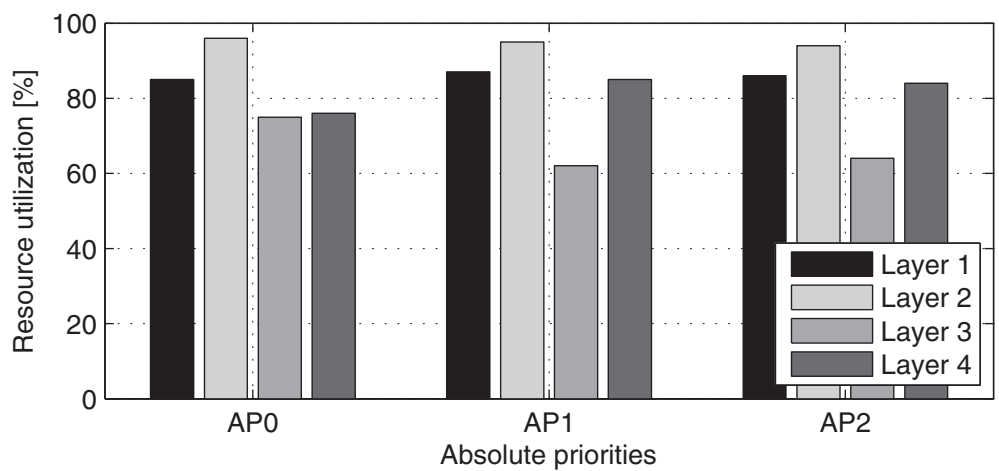

(b) $100 \%$ LTE penetration

Figure 5 Resource utilization comparison for different settings of APs. (a) 50\% LTE penetration. (b) 100\% LTE penetration. 
(e.g., $L 1$ and $L 3)$. Such a reduction of interference would mean a lower dropping ratio in the network. Conversely, the macro-offloading performed by extending the cell edge area of small cells (e.g., pico-cells) is rather limited.

Finally, Table 2 includes some other key performance indicators related to signaling and radio features. In particular, the number of reselections per second (nRes/s), the number of $\mathrm{HOs}$ per second $(\mathrm{nHO} / \mathrm{s})$, the $\mathrm{HO}$ pingpong ratio (HO PP ratio) and the radio link failure ratio (RLF ratio) are shown. The main difference in signaling cost between the proposed settings $\left(\mathrm{AP}_{1}\right.$ and $\left.\mathrm{AP}_{2}\right)$ and the reference case $\left(\mathrm{AP}_{0}\right)$ is that the number of reselections is increased by approximately $20 \%$ and $40 \%$ with $50 \%$ and $100 \%$ LTE penetrations, respectively. Regarding the radio aspect, there is no appreciable difference between settings, only that the RLF ratio is decreased by approximately $1 \%$ when the LTE penetration changes from $50 \%$ to $100 \%$.

\subsection{Simulation results of traffic steering in connected mode}

In connected mode, the parameter adjustment for traffic steering described in Algorithm 1 involves sweeping of $B 2 / 3 A$ thresholds. This study has been carried out with $\mathrm{AP}_{2}$ and $100 \%$ LTE penetration. Figure 6 shows the dropping ratio drawn from sweeping $B 2 \_1$. Following the 3GPP specifications, the ranges of valid values for LTE and HSPA are from -140 to $-44 \mathrm{dBm}$ [20] and from -120 to $-25 \mathrm{dBm}$ [21], respectively. However, in this paper, the represented ranges have been bounded since values outside the represented range lead to undesired values of the indicators. As the $B 2 / 3 A$ thresholds are assumed to be defined per layer pair, in principle, there are two different sets of parameters defined for macro-pico HOs. The $x$-axis refers to the layer pair involving layers 1 and $4(L 1$, $L 4)$, while the $y$-axis corresponds to the layer pair formed by layers 2 and $4(L 2, L 4)$. It is observed that high values of $B 2 \_1$ increase the dropping ratio since $L 4$ carries out less traffic (doing the opposite of macro-offloading). On the other hand, low values of $B 2 \_1$ also increase the dropping ratio since more UEs connected to $L 4$ stay in the cell edge experiencing very bad quality. The optimal value regarding the dropping ratio (i.e., the minimum value) is achieved by two settings: $(-104,-104 \mathrm{dBm})$ and $(-100$, $-108 \mathrm{dBm}$ ). To select a single setting, the signaling cost in terms of total number of HOs in the overall network is depicted in Figure 7 . It is noted that $(-96,-112 \mathrm{dBm})$ leads to the highest signaling cost since UEs are handed over from $L 2$ to $L 4$, and then, they are moved from $L 4$ to $L 1$, producing a strong ping-pong effect. The difference between the two selected points, $(-104,-104 \mathrm{dBm})$ and $(-100,-108 \mathrm{dBm})$, is that the former gives a reduction of $25 \%$ in signaling cost. For this reason, the optimal value is $(-104,-104 \mathrm{dBm})$. It is noted that both layer pairs have the same optimal value $\left(B 2 \_1=-104 \mathrm{dBm}\right)$ so that a single threshold $B 2 \_1$ (defined per network) could be used in this case. In addition, since the adjustments of $B 2 \_2$ and $3 A \_1$ have a minor impact from the macrooffloading perspective, these parameters are also assumed to be defined per network to simplify the optimization process (less sensitivity analysis are needed).

The following step from Algorithm 1 is the sweeping of $B 2 \_2$. Figure 8 shows the dropping ratio and the total number of HOs derived from this analysis. It is observed that increasing $B 2 \_2$ does not significantly affect the dropping ratio. This is because the inter-RAT HOs

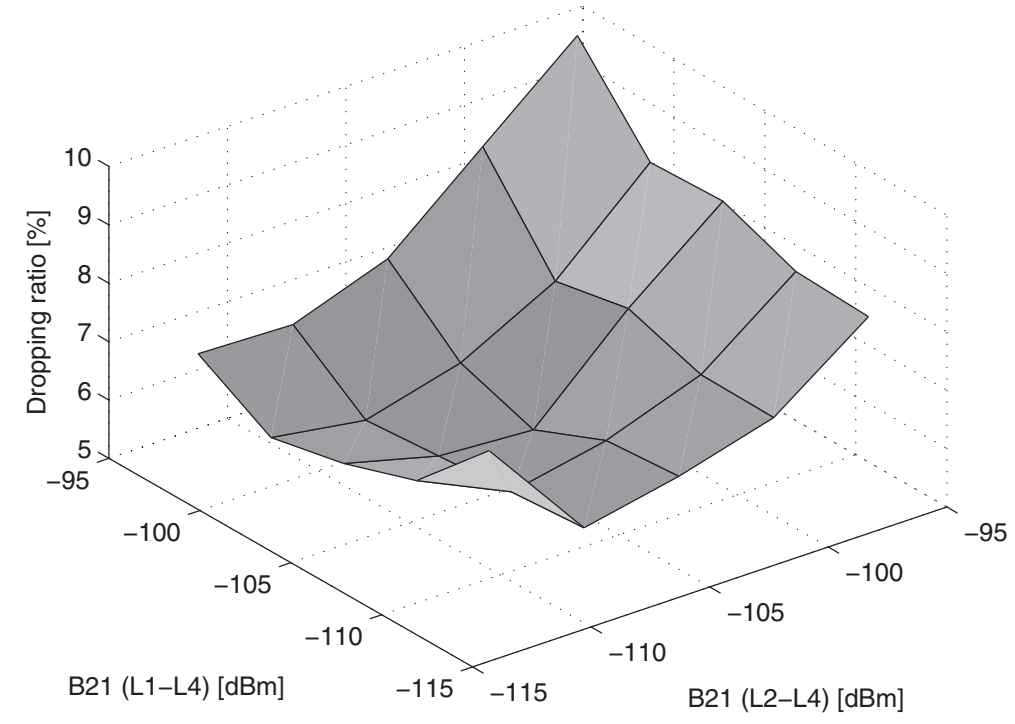

Figure 6 Dropping ratio versus threshold $B 2 \_1$. 


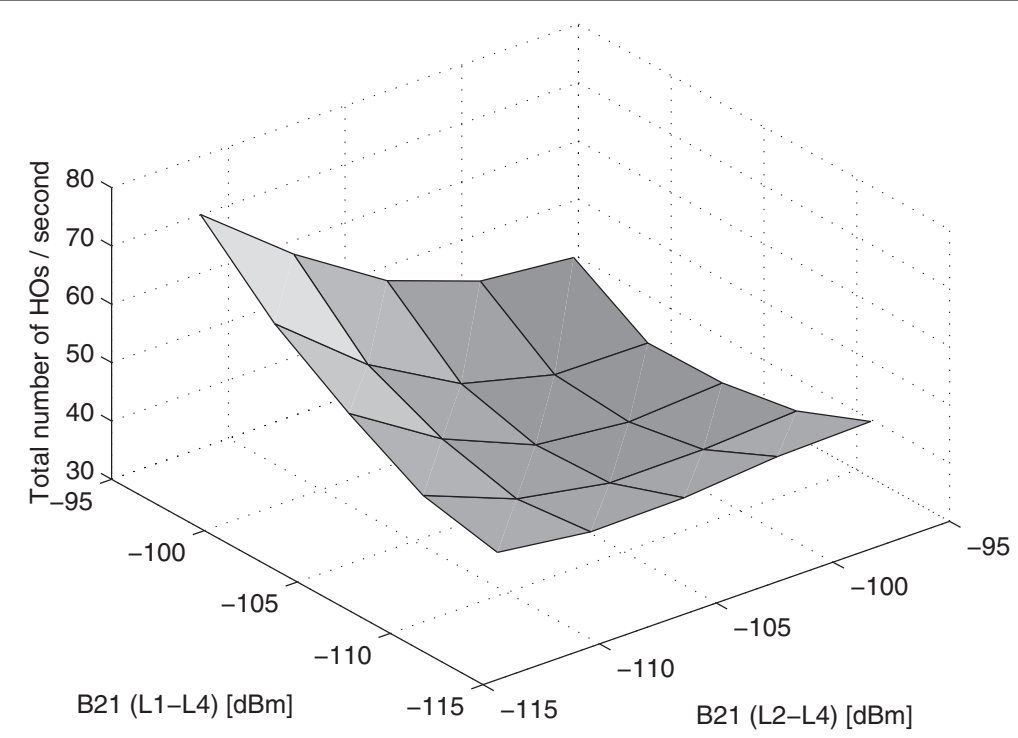

Figure 7 Total number of handover per second versus threshold B2_1.

to bad cells are being limited while macro-offloading is also being restricted so that both effects are counteracted, keeping the dropping ratio around 6\%. However, there is a minimum located at $B 2 \_2=-98 \mathrm{dBm}$. Since an acceptable signaling cost is achieved at this point (see Figure 8), B2_2 $=-98 \mathrm{dBm}$ is selected in this work.
Figure 9 shows the dropping ratio and the signaling cost referred to the sweeping of $3 A_{-} 1$. It is noted that decreasing $3 A \_1$ leads to an increment in the dropping ratio as a result of restricting the macro-offloading (the number of inter-RAT HOs is decreased), especially in pico-cells close to the macro-cell base station. For this reason, $3 A \_1=-$ $92 \mathrm{dBm}$ is the selected value since the macro-offloading is

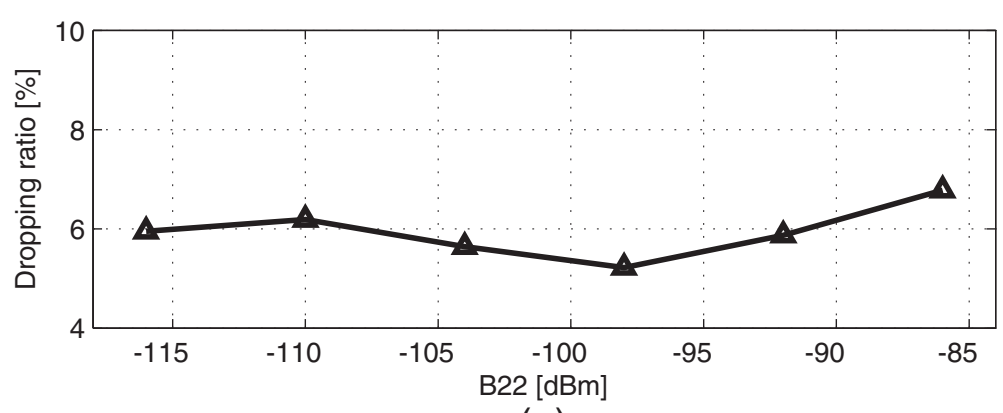

(a)

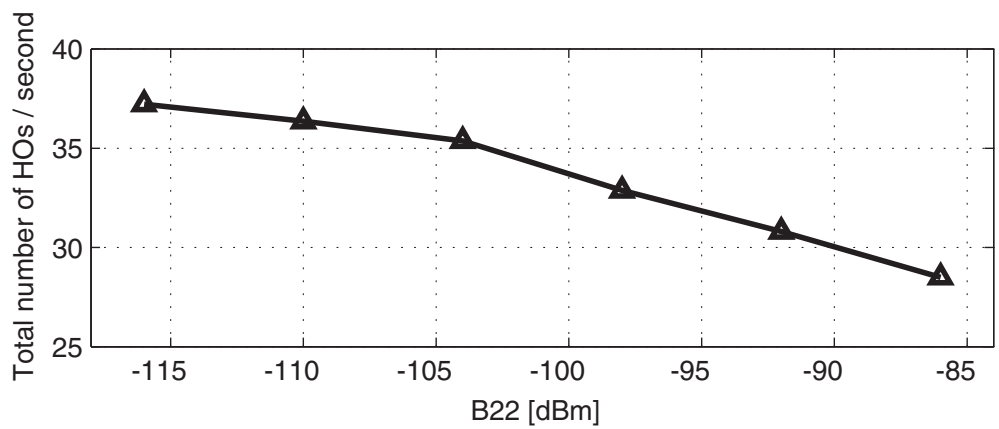

(b)

Figure 8 Performance evaluation of sweeping B2_2. (a) Dropping ratio versus B2_2. (b) Total nHO/s versus B2_2. 


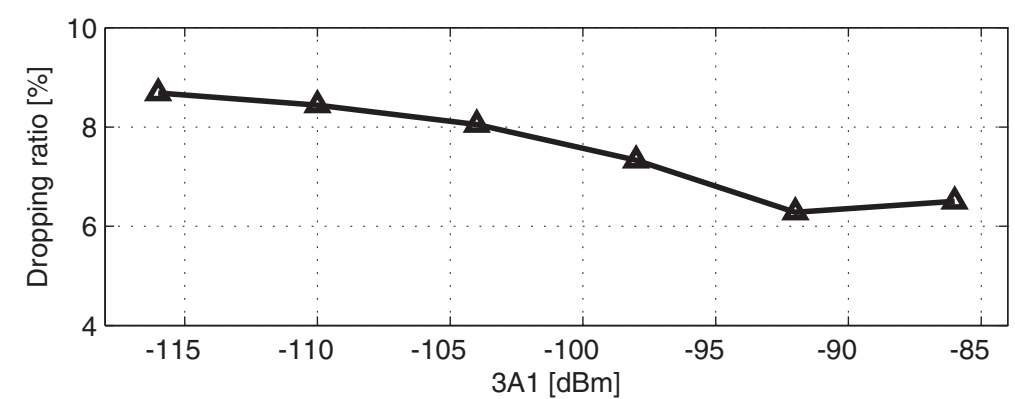

(a)

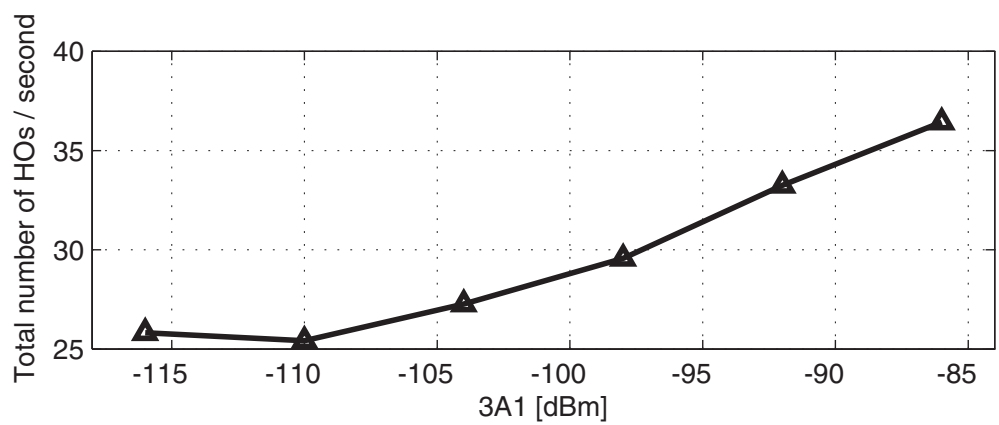

(b)

Figure 9 Performance evaluation of sweeping $3 \boldsymbol{A}_{-} \mathbf{1}$. (a) Dropping ratio versus 3A_1. (b) Total nHO/s versus 3A_1.

not affected (dropping ratio does not increase) and a $10 \%$ signaling reduction (compared to $+\infty$ ) is obtained.

Once the $B 2 / 3 A$ thresholds have been optimized, performance is assessed and compared with reference cases. The first reference case implements inter-RAT HOs based on the 3GPP $A 3$ event (neighbor cell becomes offset better than serving cell) with an offset equal to $4 \mathrm{~dB}$ and the measurement type for triggering $A 3$ is based on the signal level. It is noted that $A 3$ event has been typically used for intra-RAT HOs. To use $A 3$ event for inter-RAT HOs as a reference case, the transmitted power is assumed to be the same for both HSPA and LTE in the simulations. The second reference case also uses the $A 3$ event for inter-RAT HOs, but the measurement type is based on the signal quality. Figure 10 shows the carried traffic per layer and macro-cell area. As observed, the use of $A 3$ event leads to lower system capacity (i.e., higher dropping ratio) since more traffic is carried through $L 3$ instead of $L 4$. As $L 1$ causes co-channel interference with $L 3$, these configurations lead to worse performance. More specifically, the worst case leading to the lowest macro-offloading is ' $A 3$ level'. If the signal quality (RSRQ / Ec/No) is used

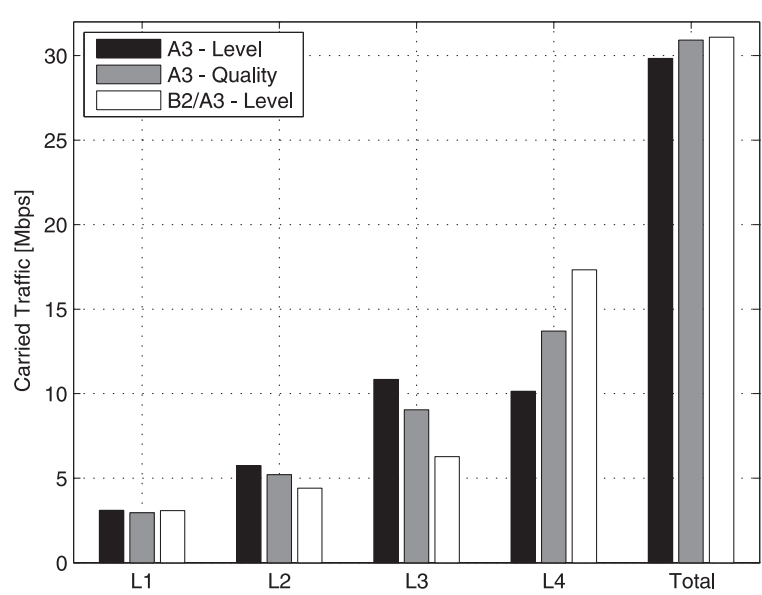

Figure 10 Carried traffic per layer for different settings of inter-RAT HOs. 
Table 3 Key performance indicators for different $\mathrm{HO}$ settings in connected mode

\begin{tabular}{|c|c|c|c|c|c|}
\hline Configuration & nRes/s & $\mathrm{nHO} / \mathrm{s}$ & $\begin{array}{l}\text { HO PP } \\
\text { ratio }\end{array}$ & $\begin{array}{l}\text { RLF } \\
\text { ratio }\end{array}$ & $\begin{array}{c}\text { Dropping } \\
\text { ratio }\end{array}$ \\
\hline A3 level & 152.4 & 63.8 & 9.2 & 2.5 & 13.4 \\
\hline A3 quality & 188.9 & 41.9 & 2.7 & 2.6 & 7.4 \\
\hline B2/3A level & 192.8 & 29.3 & 6.8 & 6.7 & 5.4 \\
\hline
\end{tabular}

Total number of UEs $=2,100$. nRes/s, number of reselections per second; $\mathrm{nHO} / \mathrm{s}$, number of HOs per second; $\mathrm{HO} \mathrm{PP}$ ratio, $\mathrm{HO}$ ping-pong ratio; $\mathrm{RLF}$ ratio, radio link failure ratio.

instead, the macro-offloading is higher (especially for $L 2$ ) since this measurement type is load-sensitive, i.e., due to the RSRQ and Ec/No definition, such indicators also depend on the load in the serving cell so that pico-cells automatically perform cell range extension when many resources are available. Conversely, the proposed setting provides the highest carried traffic since the $B 2 / 3 A$ thresholds have been thoroughly adjusted to offload traffic towards L4. It is noted that not only the macrooffloading is higher (e.g., $L 2$ carries less traffic) but also the traffic distribution between pico-layers is different, i.e., less traffic is carried by $L 3$ to avoid interfering with $L 1$.

Table 3 shows the main key performance indicators to compare the proposed configuration $(B 2 / 3 A$ level) with the reference cases. As a result of a better UE distribution across the layers, the proposed configuration achieves the lowest dropping ratio. Regarding the signaling cost, it is noted that the number of HOs is greatly decreased (30\%) compared to the second reference case, while the number of reselections is slightly increased (2\%). Thus, the proposed configuration significantly reduces the signaling cost in connected mode, where signaling is more critical. Lastly, regarding the radio aspect, it is observed that the proposed configuration increases the RLF ratio as a result of the pico-cell range extension, which increases the number of UEs in the cell edge, where the connection quality is worse.

\section{Conclusions}

This paper has analyzed two traffic steering techniques in an HSPA/LTE multi-layer wireless network. The purpose of these techniques is to offload as much traffic as possible from the macro-layer to the pico-layer since extra capacity is available in pico-cells. To achieve this objective, the absolute priorities of the cell reselection algorithm in idle mode and the thresholds of $B 2 / 3 A$ events for inter-RAT HOs in connected mode have been statically modified to further offload the macro-layer. In addition, an algorithm to simplify the task of adjusting the $B 2 / 3 A$ thresholds when the measurement type is based on the signal level has been proposed.

Simulation results show that user distributions in idle and connected mode can be controlled by adjusting the APs, although the macro-offloading performed is very limited, even with $100 \%$ LTE penetration in the UEs. The user distributions between the two co-sited pico-layers are more sensitive to APs, which means that more traffic (approximately 1.7 Mbps) can be transferred from HSPA to LTE with 50\% LTE penetration and approximately 3 Mbps with 100\% LTE penetration. The main benefit of adjusting APs is that the HSPA pico-layer generates lower interference to the co-channel HSPA macro-layer, decreasing the dropping ratio in the network. This is especially observable with $50 \%$ LTE penetration, where the LTE pico-cells are typically less loaded.

In the case of performing traffic steering in connected mode, the proposed algorithm for adjusting the $B 2 / 3 A$ thresholds allows to control the user distribution in a more accurate way than the reference cases based on the $A 3$ event, which means that further offload traffic from macro-cells can be achieved. In addition, a significant amount of traffic can be transferred from HSPA picocells to the LTE pico-layer, thus, reducing the interference that the HSPA pico-layer causes to the macro-layer and increasing the total carried traffic. More specifically, the benefits of this technique are lower dropping ratio (up to $8 \%$ decrease) and lower signaling cost ( $30 \%$ decrease). The main drawback is an increase of the radio link failure (4\%), as a result of the cell range extension.

\section{Abbreviations \\ 3GPP: 3rd Generation Partnership Project; AP: absolute priority; GSM: Global System for Mobile Communications; HetNet: heterogeneous network; HO: handover; HO PP: handover ping-pong; HSPA: high-speed packet access; LTE: long-term evolution; LTE-A: long-term evolution-advanced; $\mathrm{nHO}$ : number of handovers; nRes: number of reselections; RAT: radio access technology; RLF: radio link failure; RSCP: received signal code power; RSRP: reference signal received power; RSRQ: reference signal received quality; UE: user equipment; UMTS: Universal Mobile Telecommunications System.}

\section{Competing interests}

The authors declare that they have no competing interests.

\section{Acknowledgements}

This work has partially been supported by Junta de Andalucía (Excellence Research Program, project P08-TIC-4052).

\section{Author details}

${ }^{1}$ Communications Engineering Department, University of Málaga, Malaga 29071, Spain. ${ }^{2}$ Nokia Siemens Networks, Aalborg, Denmark. ${ }^{3}$ Aalborg University, Aalborg 9220, Denmark.

Received: 6 November 2012 Accepted: 25 April 2013

Published: 20 May 2013 


\section{References}

1. HJ Wang, RH Katz, J Giese, in Proceedings of IEEE Workshop on Mobile Computing Systems and Applications (WMCSA '99). Policy-enabled handoffs across heterogeneous wireless networks (IEEE Piscataway, 1999), pp. 51-60

2. X Gelabert, J Pérez-Romero, O Salient, R Agustí, in Proceedings of IEEE 64th Vehicular Technology Conference (VTC). 4-dimensional Markov model for the evaluation of radio access technology selection strategies in multiservice scenarios (IEEE Piscataway, 2006), pp. 1-5

3. L Giupponi, R Agustí, J Pérez-Romero, O Salient, A framework for JRRM with resource reservation and multiservice provisioning in heterogeneous networks. Mobile Netw. Appl. 11, 825-846 (2006)

4. L Giupponi, R Agustí, J Pérez-Romero, O Salient, in Proceedings of the 6th IEEE International Conference on $3 \mathrm{G}$ and Beyond. A fuzzy neural joint radio resource management in a multi-cell scenario supporting a multiservice architecture (IEEE Piscataway, 2007), pp. 1-5

5. A Furuskar, J Zander, Multiservice allocation for multiaccess wireless systems. IEEE Trans. Wireless Commun. 4, 174-184 (2005)

6. R Nasri, A Samhat, Z Altman, in Proceedings of IEEE International Symposium on a World of Wireless, Mobile and Multimedia Networks. A new approach of UMTS-WLAN load balancing; algorithm and its dynamic optimization (IEEE, 2007), pp. 1-6

7. A Pillekeit, F Derakhshan, E Jugl, A Mitschele-Thiel, in Proceedings of IEEE 60th Vehicular Technology Conference (VTC), vol. 6. Force-based load balancing in co-located UMTS/GSM networks (IEEE Piscataway, 2004), pp. 4402-4406

8. S Horrich, S Ben Jamaa, P Godlewski, in Proceedings of the Third International Conference on Wireless and Mobile Communications (ICWMC). Adaptive vertical mobility decision in heterogeneous networks (IEEE, 2007), p. 44

9. D Turina, A Furuskar, in Proceedings of the 8th International Conference on Telecommunications (ConTEL), vol. 1. Traffic steering and service continuity in GSM-WCDMA seamless networks (IEEE Piscataway, 2005), pp. 77-82

10. NTK Jorgensen, D Laselva, J Wigard, in Proceedings of IEEE 73rd Vehicular Technology Conference (VTC). On the potentials of traffic steering techniques between HSDPA and LTE (IEEE Piscataway, 2011)

11. 3GPP. Evolved Universal Terrestrial Radio Access (E-UTRA) and Evolved Universal Terrestrial Radio Access Network (E-UTRAN); Overall description; Stage 2, version 9.1.0 (2009-09). TS 36.300 (3GPP Valbonne, 2009)

12. I de la Bandera, S Luna-Ramírez, R Barco, F Ruiz, M Toril, M Fernández-Navarro, in Proceedings of the 5th International Conference on Broadband Communications and Biomedical Applications, IB2COM. Inter-system cell reselection parameter auto-tuning in a joint-RRM scenario (IEEE Piscataway, 2010), pp. 1-6

13. S Luna-Ramírez, M Toril, F Ruiz, M Fernández-Navarro, in Proceedings of the 14th IEEE Mediterranean Electrotechnical Conference, MELECON. Adjustment of a fuzzy logic controller for IS-HO parameters in a heterogeneous scenario (IEEE Piscataway, 2008), pp. 29-34

14. S Hamalainen, H Sanneck, C Sartori, LTE Self-Organising Networks (SON): Network Management Automation for Operational Efficiency. (Wiley, Hoboken, 2012)

15. 3GPP. Evolved Universal Terrestrial Radio Access (E-UTRA) User Equipment (UE) procedures in idle mode, version 10.3 .0 (2011-09), TS 36.304 (3GPP Valbonne, 2011)

16. 3GPP. Evolved Universal Terrestrial Radio Access (E-UTRA) Radio Resource Control (RRC); Protocol specification, version 9.0.0 (2009-09), TS 36.331 (3GPP Valbonne, 2009)

17. 3GPP. Technical Specification Group Radio Access Network; Radio Resource Control (RRC); Protocol Specification, version V11.0.0 (2011-12). TS 25.331 (3GPP Valbonne, 2009)

18. M Halgamuge, $\mathrm{H} \mathrm{Vu}, \mathrm{K}$ Ramamohanarao, M Zukerman, A call quality performance measure for handoff algorithms. Int. J. Commun. Syst. 24 363-383 (2011)
19. I Viering, M Döttling, A Lobinger, in Proceedings of International Conference on Communications (ICC'09). A mathematical perspective of Self-Optimizing Wireless Networks (IEEE Piscataway, 2009), pp. 1-6

20. 3GPP. Evolved Universal Terrestrial Radio Access (E-UTRA); Requirements for support of radio resource management, version 9.1.0 (2009-09), TS 36.133 (3GPP Valbonne, 2009)

21. 3GPP. Requirements for support of radio resource management (FDD), version 11.3.0 (2012-12), TS 25.133 (3GPP Valbonne, 2012)

doi:10.1186/1687-1499-2013-133

Cite this article as: Muñoz et al:: Adjustment of mobility parameters for traffic steering in multi-RAT multi-layer wireless networks. EURASIP Journal on Wireless Communications and Networking 2013 2013:133.

\section{Submit your manuscript to a SpringerOpen ${ }^{\mathcal{O}}$ journal and benefit from:}

- Convenient online submission

- Rigorous peer review

- Immediate publication on acceptance

- Open access: articles freely available online

- High visibility within the field

- Retaining the copyright to your article

Submit your next manuscript at $\gg$ springeropen.com 